\title{
Scanning Transmission X-Ray Microscopy as a Novel Tool to Probe Colloidal and Photonic Crystals
}

\author{
Matti M. van Schooneveld, Jan Hilhorst, Andrei V. Petukhov, Tolek Tyliszczak, \\ Jian Wang, Bert M. Weckhuysen, Frank M. F. de Groot, * and Emiel de Smit*
}

\begin{abstract}
Photonic crystals consisting of nano- to micrometer-sized building blocks, such as multiple sorts of colloids, have recently received widespread attention. It remains a challenge, however, to adequately probe the internal crystal structure and the corresponding deformations that inhibit the proper functioning of such materials. It is shown that scanning transmission X-ray microscopy (STXM) can directly reveal the local structure, orientations, and even deformations in polystyrene and silica colloidal crystals with 30-nm spatial resolution. Moreover, STXM is capable of imaging a diverse range of crystals, including those that are dry and inverted, and provides novel insights complementary to information obtained by benchmark confocal fluorescence and scanning electron microscopy techniques.
\end{abstract}

\section{Introduction}

The self-assembly of monodisperse colloidal particles is a promising method for fast and cheap production of photonic materials. ${ }^{[1-4]}$ These materials hold promise for applications in telecommunications, solar-energy harvesting, and

M. M. van Schooneveld, Prof. B. M. Weckhuysen, Prof. F. M. F. de Groot, Dr. E. de Smit

Inorganic Chemistry \& Catalysis

Debye Institute for Nanomaterials Science

Utrecht University

Sorbonnelaan 16, 3584 CA Utrecht, The Netherlands

E-mail: F.M.F.deGroot@uu.nl; E.deSmit@gmail.com

J. Hilhorst, Dr. A. V. Petukhov

Van 't Hoff Laboratory for Physical \& Colloid Chemistry

Debye Institute for Nanomaterials Science

Utrecht University

Padualaan 8, 3584 CH Utrecht, The Netherlands

Dr. T. Tyliszczak

Advanced Light Source

Lawrence Berkeley National Laboratory

Berkeley, CA, 94720, USA

Dr. J. Wang

Canadian Light Source

University of Saskatchewan

Saskatoon, SK, S7N OX4, Canada

DOI: 10.1002/smll.201001745 low-threshold lasing. ${ }^{[5]}$ The convective assembly technique ${ }^{[1-4]}$ is a well-established method to produce macroscopic colloidal crystals with a face-centered cubic (fcc) structure. Colloidal crystals obtained in this manner can be used as a template for making inverted photonic crystals of materials with a desired refractive index. ${ }^{[2,6]}$ The inverted structure can then serve as a photonic crystal. In order to obtain crystals with a full photonic bandgap, however, the fcc structure needs to be controlled since the photonic bandgap is highly sensitive to stacking faults, dislocations, and other deformations. ${ }^{[7]}$ To achieve perfect crystals, detailed knowledge about the crystalgrowth mechanism and resulting defect structures is required. The growth mechanism has received widespread attention over the last decade, ${ }^{[4,8,9]}$ resulting in the proposal of several growth models, but experimental evidence supporting these models is lacking. Moreover, the defect structures themselves have hardly been investigated and the few reported studies indicate that the dominant fcc crystals still possess a significant degree of disorder resulting in disadvantageous effects on the properties of photonic crystals. ${ }^{[10,11]}$

A serious obstacle in the study of defect structures is the absence of appropriate techniques that probe the internal structure of photonic crystals in three dimensions. The most widely applied technique is scanning electron microscopy (SEM) but this technique is inherently surface specific. The only possibility for studying parts of the crystal internal structure by SEM is through physically cutting crystals apart 
and studying their cut edges. ${ }^{[1]}$ This, however, introduces the risk of modifying the crystal structure in the cutting process. The application of transmission electron microscopy (TEM) to the study of these materials is limited since the technique's probing depth is a few hundred nanometers at best. Another commonly applied technique is confocal scanning laser microscopy (CSLM). CSLM is excellent for the in situ investigation of immersed, fluorescent, and refractive-indexmatched colloidal crystals with particle diameters on the order of a micrometer. ${ }^{[12]}$ However, convectively assembled crystals and their inverted crystals are dry and in contact with air, which implies that the structures and their surroundings are not refractive-index matched. CSLM imaging is then restricted to the first one or two crystal layers, also preventing the study of the crystal internal structure. One way to overcome this is by infiltrating the inverted crystal with a refractiveindex-matching fluid before imaging the structure ${ }^{[13]}$ but capillary forces acting on the crystal during this process may very well change the structure, resulting in unreliable characterization. In addition, CSLM has the disadvantage that many particle sizes used for colloidal crystals are too small to be imaged, although, for example, the recently developed stimulated emission depletion (STED) microscopes may circumvent this problem. ${ }^{[14]}$ Alternatively, the internal crystal structure may be studied in reciprocal space by small-angle $\mathrm{X}$-ray diffraction (SAXD), ${ }^{[11,15,16]}$ which is a powerful tool to investigate the crystal structure and planar defects on large length scales, but the local structure important to crystal growth is also unresolvable by this technique.

In this Full Paper, we present the first study of convectively assembled colloidal crystals by scanning transmission X-ray microscopy (STXM). The technique uses soft X-rays $(E<2 \mathrm{keV})$ that have a smaller penetration depth than hard X-rays $(E>2 \mathrm{keV})$, used in, for example, the recently proposed highresolution transmission X-ray microscopy (HRTXM). ${ }^{17]}$ However, STXM has the advantage that an even-higher spatial resolution is accessible, richer chemical information can be obtained due to a superior energy resolution, and lighter elements can be studied at their specific X-ray absorption edges, such as the carbon $\mathrm{K}$ - and silicon $\mathrm{K}$-edge in the here-presented polystyrene (PS) and silica $\left(\mathrm{SiO}_{2}\right)$ colloidal crystals. Compared to coherent $\mathrm{X}$-ray diffraction imaging (CDI), the characterization of local defect structures is more straightforward in STXM since it is real-space X-ray microscopy. Our results illustrate that STXM offers information on the internal, local structure of such crystals, inaccessible by and complementary to the techniques mentioned above. We end with a detailed comparison of STXM, CSLM, and SEM imaging for the study of photonic and colloidal materials.

\section{Results and Discussion}

Polystyrene colloids with a mean diameter of $194 \mathrm{~nm}$ and a polydispersity of $3.7 \%$ were synthesized according to standard literature procedures. ${ }^{[18]}$ Silica colloids were synthesized by the Stöber method (492-nm diameter; 3.2\% polydispersity) and subsequently coated with 3-methacryloxypropyltrimethoxysilane. ${ }^{[19]}$ Colloidal crystals were grown on 100-nm-thick silicon nitride windows by the convective assembly technique and SEM images of the studied crystals can be found in the Supporting Information (SI) and Figure S1 therein. ${ }^{[1]}$ The thin silicon nitride windows are almost X-ray transparent and, as such, prevent significant attenuation of the X-ray signal by the substrate. Further experimental details of the colloid synthesis and convective assembly of the crystals can be found in the Experimental Section. The polystyrene and silica colloidal crystals were subsequently studied along their physical edges with interferometercontrolled STXM. ${ }^{[20-22]}$ Figure 1a shows a schematic representation of the STXM set-up, in which a monochromatic X-ray bundle is focused on the sample through a Fresnel zone plate. The zone plate is a circular diffraction grating, which acts as a concave lens by diffracting X-rays at a series of alternate opaque (gold) and transparent (silicon nitride) rings. ${ }^{23,24]}$ The size of the resulting beam spot is mainly determined by the distance between the two outer gold rings within the zone plate, provided that the rest of the optics, such as the order-sorting aperture and slit sizes are well aligned and set. Here, a zone plate with an outermost zone width, $\Delta r$, of $25 \mathrm{~nm}$ was used, limiting the Rayleigh-criterion spatial resolution

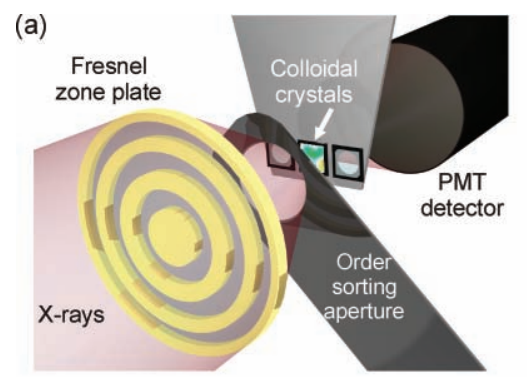

(c)

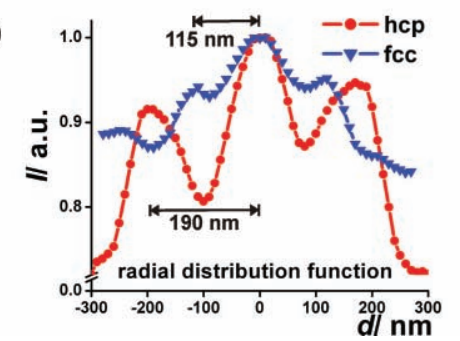

(b)
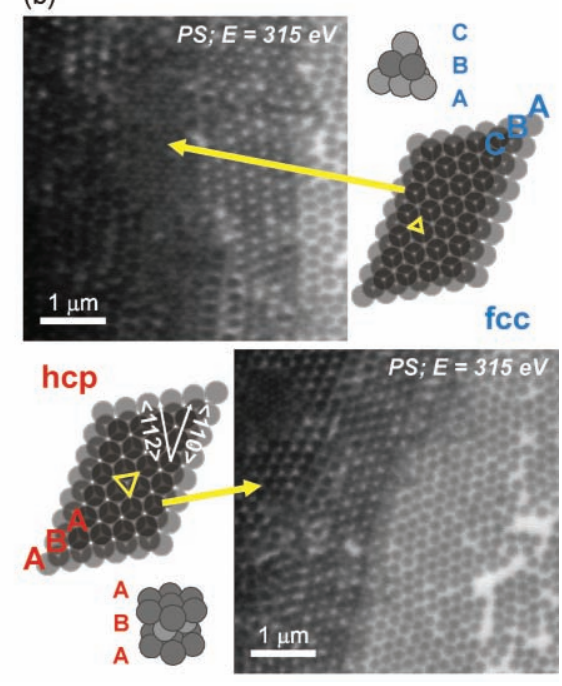

Figure 1. a) Schematic representation of the STXM set-up. Monochromatic X-rays are focused through a Fresnel zone plate on the colloidal crystal. The transmission of X-rays is detected with a photomultiplier tube (PMT). b) Two X-ray transmission images acquired at $315 \mathrm{eV}$ on the carbon K-edge show the presence of one (right side) to three (left side) polystyrene (PS) colloidal layers. The transmission spatial profile is highly different for fcc and hexagonal closepacked (hcp) crystal structures, as indicated in the upper and lower panels, respectively. Note that the $\langle 110\rangle$ and $\langle 112\rangle$ directions are indicated in the hcp crystal. c) Radial distribution functions of the most X-ray intense points in the three-layered regions of the panels in (b) indicate that the distance, $d$, between the most transparent regions is $190 \mathrm{~nm}$ (red curve) and $115 \mathrm{~nm}$ (blue curve) for the hcp and fcc structures, respectively. 
$\Delta r_{\text {Rayleigh }}$ to $30 \mathrm{~nm}\left(\Delta r_{\text {Rayleigh }} \approx 1.22 \cdot \Delta r\right)$ for the presented data. The depth of focus, $\Delta z$, is given by $\pm 2 \Delta r^{2} / \lambda$ and is thus theoretically $\approx 600 \mathrm{~nm}$ at $300 \mathrm{eV}$ and $\approx 3700 \mathrm{~nm}$ at $1845 \mathrm{eV}$, the typical photon energies used here. ${ }^{[23]}$ By measuring $\mathrm{X}$-ray absorption at varying positions and energies, it is possible to acquire a spectral image of a region of interest in which every pixel of an image contains an X-ray absorption spectrum that allows detailed chemical specification (for example the type of element, oxidation state, and coordination number can be deduced). ${ }^{[25,26]}$

A single-energy X-ray transmission image can, however, contain novel information by itself, as shown in Figure 1b (see the Experimental Section for detailed STXM setup and acquisition details). Two pictures taken at $315 \mathrm{eV}$ are shown of two different spots in the same polystyrene colloidal crystal. The images were taken above the carbon K-edge and a large part of the revealed absorption is thus specifically due to the carbon present in polystyrene $\left(\left(-\mathrm{C}_{8} \mathrm{H}_{8}-\right)_{n}\right)$. A single layer of colloids is clearly resolved on the right side of both images, while multiple colloidal layers were found towards the left of the micrographs. First, a double colloidal layer, which is similar in both images, was observed. A third layer revealed an fcc and a hexagonal close-packed (hcp) crystal structure in the two different images. The fcc and hcp stackings differ in the way the subsequent colloidal layers are positioned atop of each other. A hcp phase has an ABA stacking, in which the third colloidal layer is located directly above the first, while the fcc phase has an ABC stacking, in which the third layer has a different position from both the first and second layers. This yields crystal structures that are completely closed (fcc) and partly open (hcp) in the direction perpendicular to the three colloidal layers, as depicted in the schematic representations in Figure 1b. The difference between the open and closed structures can be readily observed in the X-ray transmission images, where the open holes are more transparent than the most transparent, but closed, parts of the fcc structures. Also, the theoretical distance between the most-transparent regions in a hcp structure (the open holes) is equal to the particle diameter $\sigma$, while the most-transparent (but closed) points are $\sigma / \sqrt{3}$ apart in fcc structures. Figure 1c shows the radial distribution functions of the most X-ray transparent points in the hcp and fcc-packed regions of the images. The radial distribution functions were obtained using the aXis2000 STXM dataprocessing software ${ }^{[27]}$ and give the average distance from one hole in the colloidal layer to its nearest neighbor hole for hcp (and from one most-transparent region to another in fcc). Indeed, it was deduced that the average length between these most transparent regions is 190 and $115 \mathrm{~nm}$ in the hcp and fcc structures, respectively, which match the theoretical values of $\sigma=194 \mathrm{~nm}$ in the hcp and $\sigma / \sqrt{3}=112 \mathrm{~nm}$ in the fcc structures. Both the differences in absolute intensity in the crystal layers and the distances between the most transparent regions allow for the assignment of the crystal structure being hcp or fcc. Such determination of the type of crystal structure from a single transmission image, as demonstrated here for three colloidal layers, is not feasible with any of the other current techniques for the study of colloidal crystals.
Moreover, spatially resolved and quantitative determination of the crystal thickness is possible with STXM if an X-ray transmission image is measured below and on a crystal-element-specific X-ray absorption edge. These transmission images can both be converted into optical density $(O D)$ maps with $O D=-\ln \left(I / I_{0}\right)$, where $I_{0}$ is taken as the intensity of the image background. The difference between the $O D$ maps on and below the X-ray absorption edge yield an $O D$ map that is element specific and corrected for differences in X-ray attenuation at different energies. This map is subsequently converted into a material-thickness map using $O D=\mu \rho t$, where $\mu$ is the photoabsorption cross-section or mass-absorbance coefficient, $\rho$ is the material density, and $t$ is the material thickness. Two material-thickness maps are displayed in Figure 2a and b for the exact same crystal areas
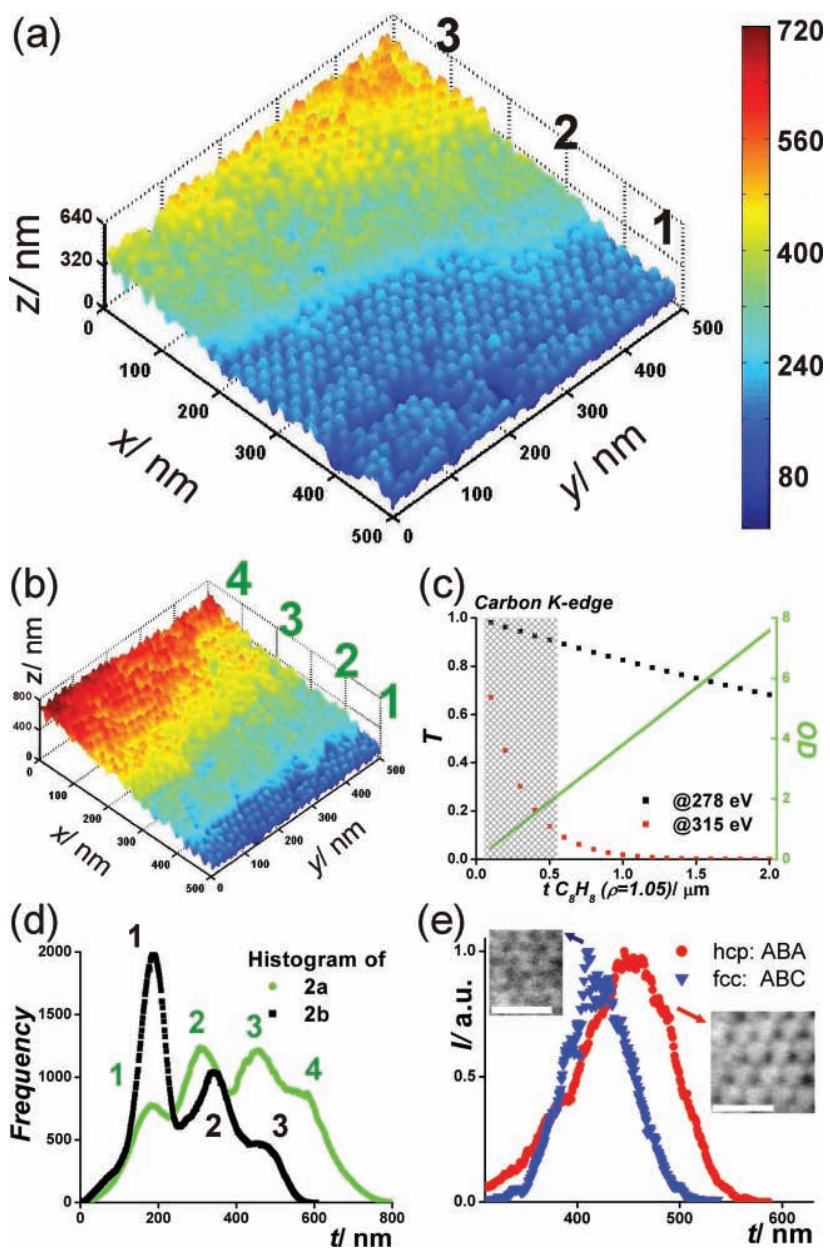

(e)

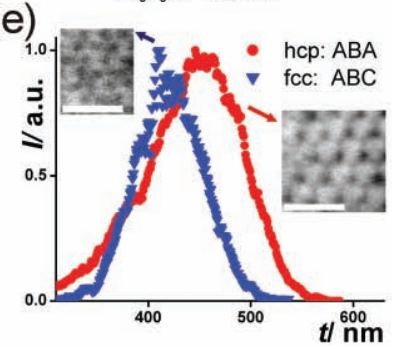

Figure 2. Thickness maps of polystyrene colloidal crystals at the same locations as shown in Figure $1 b$ indicating up to a) three and b) four colloidal layers. c) The X-ray transmittance $(T)$ below and above the carbon K-edge at 278 and $315 \mathrm{eV}$, respectively, is indicated with black and red dots. The corresponding material-specific optical density $(O D)$ is shown with a green solid line and the thickness $(t)$ regime in which the optical density scales linearly with the material thickness is indicated by the shaded area. d) Histograms of the thickness of the colloidal crystals shown in (a) and (b) with, respectively, black and green dots. e) Thickness histograms of three colloidal layers with an fcc (blue triangles) or hcp (red dots) crystal structure. The scale bar in the inset corresponds to $500 \mathrm{~nm}$. 
that were shown in Figure 1b. The images were taken below and above the carbon K-edge at 278 and $315 \mathrm{eV}$, respectively, since carbon is the principal constituent of polystyrene. Here, the polystyrene density ${ }^{[18]}$ was taken to be $1.05 \mathrm{~g} \mathrm{~cm}^{-3}$ and the mass-absorbance coefficient for polystyrene was estimated to be $36000 \mathrm{~cm}^{2} \mathrm{~g}^{-1}$ using the aXis2000 software that allows for the calculation of $\mu$ (calculate X-ray parameters SF package). Figure $2 \mathrm{c}$ shows the $\mathrm{X}$-ray transmittance at 278 and $315 \mathrm{eV}$, and the corresponding $O D$ as a function of polystyrene thickness, calculated from semi-empirical atomicscattering factors. ${ }^{[28]}$ In the regime of approximately $0.2<$ $O D<2.1$, the material thickness scales linearly with $O D$ (or $O D=\mu \rho t$ holds) and the thickness can be quantified in this regime. For polystyrene measured at the carbon K-edge, this means that the material thickness can be quantitatively determined for layers that are 50-550-nm thick. Thickness histograms of the crystals displayed in Figure $2 \mathrm{a}$ and $\mathrm{b}$ are presented in Figure $2 \mathrm{~d}$ and clearly show the presence of up to three and four colloidal layers, respectively. The quantification of the number of colloidal layers from a single map is unrivaled and cannot be done with SEM or CSLM. In addition, when regions of interest were studied that contain only three colloidal layers in a hcp or fcc stacking, the thickness histograms, as given in Figure 2e, confirm the presence of these phases. Both the thickest and the thinnest crystal parts were found in the hcp structure, as expressed in the wider thickness distribution for hcp as compared to the fcc histogram.

It becomes clear that the thickest and thinnest crystal parts are present in the hcp stacking when considering projections in hcp and fcc structures along, for example, $<112>$ and $<110>$ directions, as shown in Figure 3a and b, respectively. Note that the fcc coordinate system is used to define crystallographic directions and that the directions are indicated in Figure $1 \mathrm{~b}$ and in the insets next to the graphs. Model calculations of the crystal heights for 200-nm-diameter spheres along these directions within the two crystal types show the large variation in height within a hcp crystal compared to an fcc structure. Next to theoretical predictions, the experimentally found thickness projections along the $<112>$ and $\langle 110\rangle$ directions are shown. The experimental projections are averaged over 4-or-more line profiles that were taken from images with a short dwell time of $1 \mathrm{~ms}$ per pixel. One can appreciate that the general shape and relative intensities of the line profiles match the theoretically predicted profiles. The absolute intensities are in good agreement for the thick parts of the crystal but the thinner parts of the hcp and fcc structures appear too thick, mainly due to the limited lateral resolution. However, in Figure $3 a$, the determined thickness along the $<112>$ direction in hcp varies stronger than in fcc and even the shape of the experimental hcp $<112>$ projection shows a characteristic structure that roughly matches the theoretical one. Increasing the statistics of such line profiles by taking longer dwell times during image acquisition will significantly improve the quality of such line projections. Nevertheless, it is shown here that STXM has the capacity to discriminate between different directions within a crystal structure from $\mathrm{X}$-ray optical-density images.
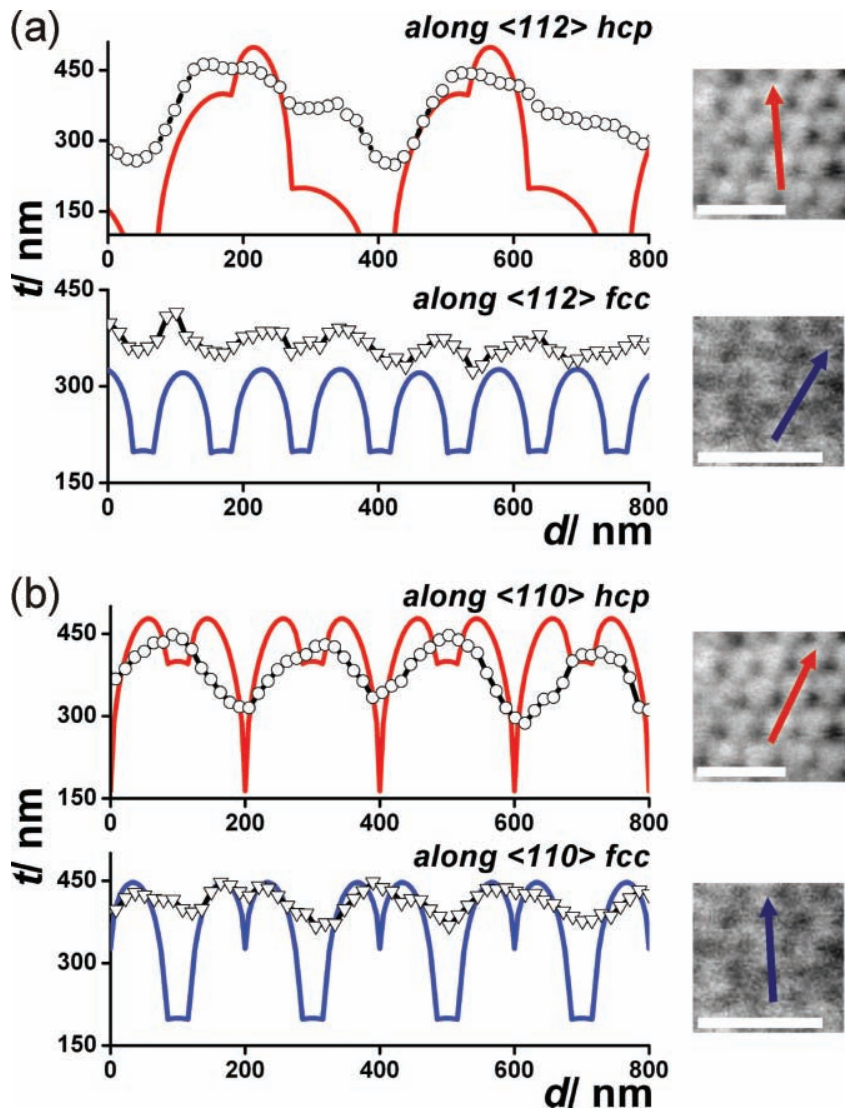

Figure 3. a) Theoretical (solid line) and experimental (symbol and solid line) thickness $(t)$ line projections along the $<112>$ direction in hcp (upper panel) and fcc (lower panel) crystal types. b) Identical projections as in (a) but along the $<110>$ direction. Note that STXM-image insets on the right correspond to the indicated crystal type and that the arrows indicate the corresponding crystal directions.

Figure 4a is not a thickness material map but a single transmission image of a large area of a silica colloidal crystal,taken on the silicon K-edge at $1845 \mathrm{eV}$. The intensity histogram of Figure 4a indicates the presence of up to 9 colloidal layers (shown as an inset) and illustrates that STXM is also capable of quantitatively imaging thicker silica-based crystals that have been proposed as photonic crystals. ${ }^{[29]}$ The application of rotation tomography could reveal the exact location of, for example, crystal deformations. Figure $4 b$ shows that the thickness of silica-based materials up to $6.5 \mu \mathrm{m}$ can be quantitatively studied at their silicon K-edge (see the SI and Figure S2 therein for a similar calculation at the silicaoxygen K-edge).

Figure 5a and $\mathrm{b}$ are exemplifications of the chemical sensitivity of STXM. A measured carbon K-edge spectrum of polystyrene is shown in Figure 5a with an energy resolution of $0.2 \mathrm{eV}$. By varying the photon energy of the microscope's light over the carbon $\mathrm{K}$-edge, the presence of transitions from $\mathrm{C} 1$ s to $\mathrm{C}=\mathrm{C} 1 \pi^{*}$ and $2 \pi^{*}$ orbitals was observed at 285 and $288.8 \mathrm{eV}$, respectively, as well as the transitions to $\mathrm{C}-\mathrm{H}^{*}$ and $\mathrm{C}-\mathrm{C} \sigma^{*}$ unoccupied molecular orbitals, which identifies the measured carbon as being present in polystyrene. ${ }^{[30]}$ The $\mathrm{X}$-ray transmission images acquired at 278 and $315 \mathrm{eV}$ respectively show that the X-ray absorption contrast is specifically 


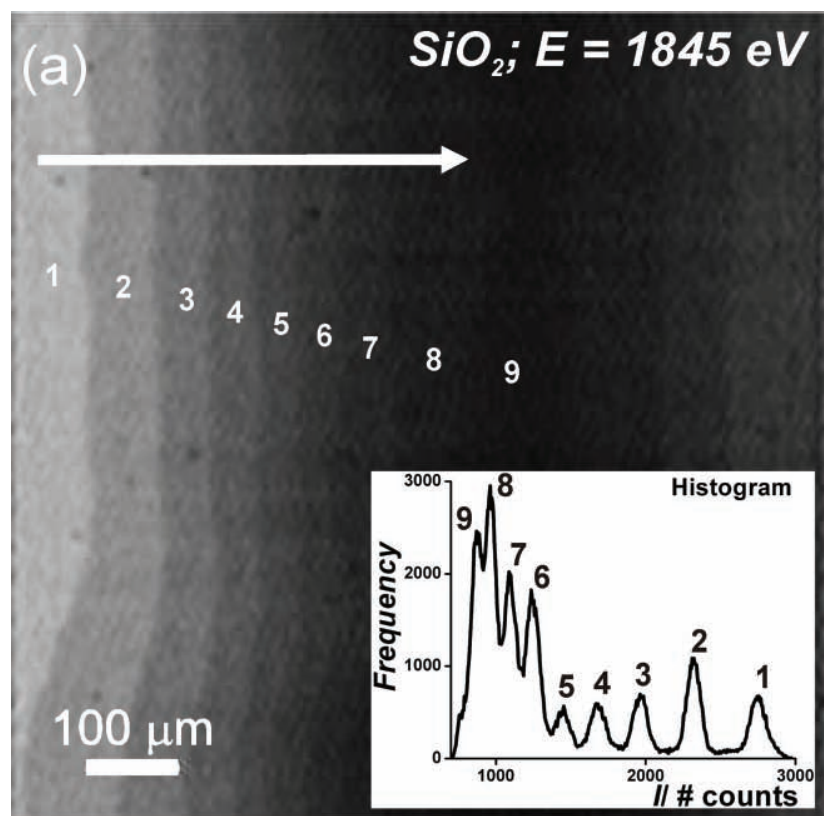

\section{(b) Silicon K-edge}

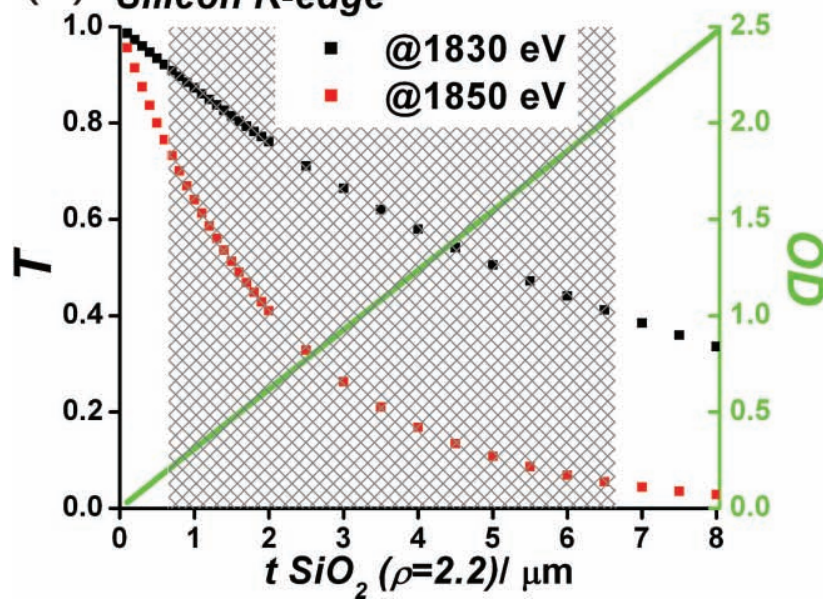

Figure 4. a) X-ray transmission image acquired at $1845 \mathrm{eV}$ on the silicon K-edge showing the presence of up to 9 colloidal silica $\left(\mathrm{SiO}_{2}\right)$ layers. The corresponding histogram is shown as an inset. The arrow indicates the direction in which the number of crystal layers on top of each other increases. b) Black and red dots indicating the transmittance $(T)$ of $\mathrm{X}$-rays through silica before and on the silicon K-edge, as a function of silica thickness $(t)$. The green solid line indicates the corresponding optical densities $(O D)$ at those thicknesses and the shaded area shows the regime where the silica thickness can be quantified.

due to the types of atoms present. Figure $5 \mathrm{~b}$ shows the silicon K-edge X-ray absorption spectrum of the amorphous silica colloids. The silicon K-edge spectrum reveals the local projected density of empty Si p orbitals ( $\mathrm{p}$ local density of states $)^{[31]}$ and the main peak at $1845.1 \mathrm{eV}$ corresponds to the $\mathrm{Si} 3 \mathrm{p}$ conduction band. In principle, the discrimination of different chemicals in every pixel, or even voxel, would allow for the study of more complex crystals that are built from more than a single constituent, like, for example, a binary ionic-colloidal crystal. ${ }^{[32]}$

As shown in the presented data, the application of STXM to the characterization of photonic and colloidal crystals can
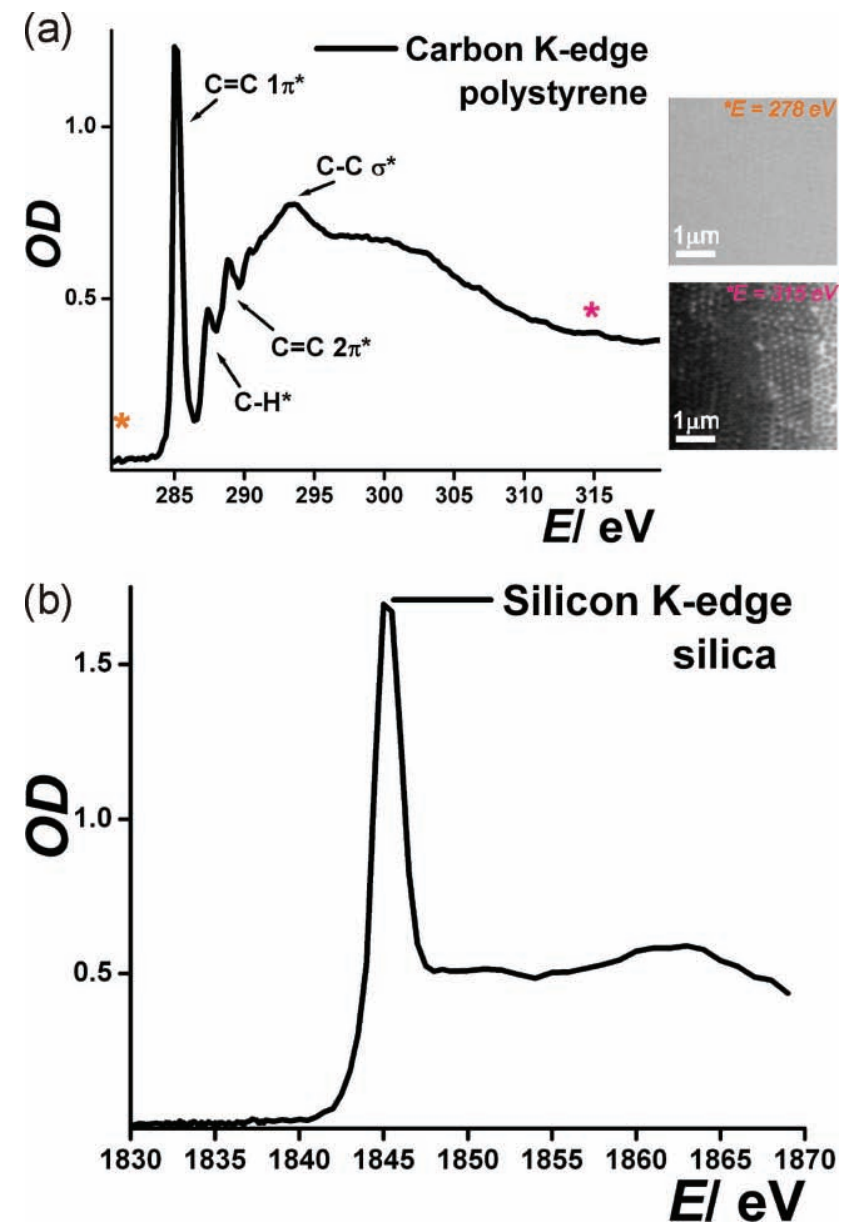

Figure 5. a) A carbon K-edge X-ray absorption spectrum of the polystyrene colloids indicating the presence of $\mathrm{C} 1 \mathrm{~s}$ to, amongst, others, $\mathrm{C}=\mathrm{C} 1 \pi^{*}$ and $2 \pi^{*}$ transitions. The STXM images on the right taken at 278 (up) and $315 \mathrm{eV}$ (down) show that the X-ray absorption contrast is specific for the carbon presence. Making use of the carbon K-edge spectral fine structure could give molecular contrast. b) The silicon K-edge X-ray absorption spectrum of the silica colloids reflects the local projected density of empty Si $p$ orbitals.

yield novel information on the local crystal structure. In order to better comprehend why STXM is favorable for this purpose, we compare the technique to CSLM and SEM, which are commonly used for the study of colloidal crystals, on the basis of three important microscope properties: the lateral Rayleigh-criterion spatial resolution, $\Delta r_{\text {Rayleigh }}$, the depth of focus, $\Delta z$ (which is related to the axial spatial resolution), and the penetration depth of the microscope's probe (photons for STXM and CSLM, electrons for SEM). A detailed comparison considering the most important technical details of each method is presented in the SI and Figure S3 therein. ${ }^{[23,33-36]}$

The most important outcomes of this comparison are summarized in Figure 6. The best obtainable lateral resolution for the techniques are $10^{-1}>10^{-2}>10^{-3} \mu \mathrm{m}$ for CSLM, STXM, and SEM, respectively. The depth of focus is very large for SEM $\left(10^{0}-10^{3} \mu \mathrm{m}\right)$ and smaller, but comparable, for CSLM and STXM $\left(10^{-1}-10^{1} \mu \mathrm{m}\right)$. SEM probes a very limited region of $10^{-3}-10^{-2} \mu \mathrm{m}$ under the material surface, while $10^{0}-10^{2}$ and $10^{-1}-10^{2}-\mu \mathrm{m}$-thick samples are accessible by CSLM and STXM, respectively. It is the unique combination of a large 


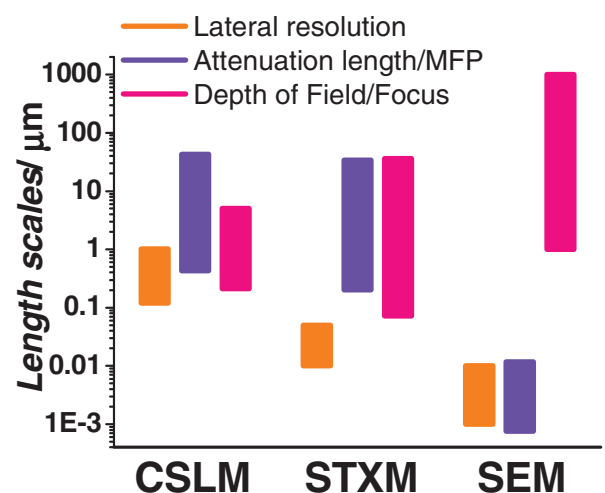

Figure 6. Characteristic length scales of three important microscope properties compared for CSLM, STXM, and SEM. The lateral resolution $\Delta r_{\text {Rayleigh }}$, the depth of focus $\Delta z$ (in CSLM and STXM) or depth of field $\Delta v$ (in SEM), and the photon-attenuation length ( $l$ in CSLM, $t$ in STXM) or electron mean free path (MFP) in SEM are compared as a function of the photon or electron energy.

and comparable depth of focus and attenuation length, in combination with an improved spatial resolution compared to CSLM, which is, moreover, independent of the incident photon energy, that makes STXM very useful for the study of colloidal and photonic crystals. Only with STXM is it possible to look through micrometer-thick materials, which are acceptably focused over their full thickness, with a lateral resolution of up to $10 \mathrm{~nm}$. This allows for the study of crystals built from relatively small building blocks. The fact that STXM does not require luminescent and refractive-index-matched materials, as is necessary for CSLM imaging, is another major advantage of the technique. This allows the internal structures of all colloidal crystals to be probed by STXM: from dry to wet crystals, from refractive-index-matched crystals to nonindex-matched inverted crystals, and from dye-containing to nonluminescent crystals. The rich chemical characterization that is feasible through the acquisition of $\mathrm{X}$-ray absorption spectra and the quantification of the material thickness are final additional STXM advantages.

Future STXM work on colloidal or photonic crystals would greatly benefit from quantitative $\mathrm{X}$-ray tomography ${ }^{[37,38]}$ by use of a rotation stage in which the beneficial, small lateral resolution and large depth of focus are fully exploited. The study of binary colloidal crystals (as, for example, shown with electron tomography for nanocrystals ${ }^{[39]}$ ), large-areaprinted colloidal layers, ${ }^{[40]}$ colloidal gels, ${ }^{[41]}$ and liquid crystals made of anisotropic particles ${ }^{[42]}$ could all benefit from STXM imaging, while the application of in situ STXM microscopy ${ }^{[43-47]}$ to study, for example, more complex colloidal phase behavior is another possibility.

\section{Conclusion}

We have shown that STXM can discriminate between fcc and hcp colloidal crystal structures from X-ray transmission images on the basis of relative intensities, the spatial distribution of such intensities, and thickness histograms of the different crystal structures. STXM is also capable of quantitatively determining the colloidal crystal thickness. Line projections within the crystal transmission images allow further for the differentiation of various crystal directions, such as, for example, the hcp $<110>$ and $<112>$ directions. X-ray absorption spectra permit the possibility of combining all of the above with rich chemical contrast for the localization of different crystal building blocks. It is the unique combination of the X-ray depth of focus and attenuation length, which can simultaneously extend to tens of micrometers, in combination with a lateral spatial resolution of up to $10 \mathrm{~nm}$ that is independent of the photon energy, that makes STXM highly favorable for the study of the local internal crystal structure over the present benchmark CSLM and SEM techniques. We foresee that STXM can play a major role in the elucidation of problems concerning colloidal and photonic crystal disorder and deformations, which up to now frequently hamper the proper functioning of such materials.

\section{Experimental Section}

Materials: Potassium persulphate (KPS; $99+\%)$ was obtained from Acros Organics, sodium dodecyl sulphate (SDS; specially pure, $>99 \%$ ) from Brunswig, styrene (for synthesis, >99\%) from Merck, and divinylbenzene (technical grade, 55\%) and vinyl acetate (>99\%) from Aldrich. Millipore water (resistivity: $18 \mathrm{M} \Omega \mathrm{cm}$ ) was used and 100-nm-thick silicon nitride $\left(\mathrm{Si}_{3} \mathrm{~N}_{4}\right)$ windows were obtained from Silson Ltd.

Synthesis of Polystyrene and Silica Colloids and Convectively Assembled Colloidal Crystals: Polystyrene seed particles with a crosslinking density of $3 \mathrm{wt} \%$ divinylbenzene were synthesized by emulsion polymerization as described by Mock et al. ${ }^{[18]}$ In short, water $(400 \mathrm{~mL})$ was heated to $80^{\circ} \mathrm{C}$ in a round-bottom flask $(1 \mathrm{~L})$. Subsequently, styrene $(50 \mathrm{~mL})$ and aqueous SDS solution $\left(100 \mathrm{~mL} ; 5 \mathrm{~g} \mathrm{~L}^{-1}\right)$ were added, followed by divinylbenzene cross-linker $(1.39 \mathrm{~mL})$. The reaction mixture was allowed to equilibrate for $1 \mathrm{~h}$, before adding KPS initiator aqueous solution ( $\left.75 \mathrm{~mL} ; 20.67 \mathrm{~g} \mathrm{~L}^{-1}\right)$. The reaction was kept at $80^{\circ} \mathrm{C}$ for $24 \mathrm{~h}$. The particles were subsequently coated with vinyl acetate in order to render them more hydrophilic. Therefore, the seed solution $(200 \mathrm{~mL})$ was heated to $80{ }^{\circ} \mathrm{C}$ for $1 \mathrm{~h}$, after which vinyl acetate $(1.70 \mathrm{~mL})$ was added in four aliquots $(0.425 \mathrm{~mL})$ with 15 -min intervals. Directly after the first addition, aqueous KPS solution (5.05 mL; 0.67 wt\%) was added. After the final addition, the reaction was allowed to continue for $24 \mathrm{~h}$. Particles were purified at least three times by centrifugation and subsequent redispersion steps before use. The average colloid diameter was determined to be $194 \mathrm{~nm}$ with a polydispersity of $3.7 \%$ (defined as the standard deviation over the mean size; $n>200$ ) by TEM measurements on a Philips Tecnai 12 operated at $120 \mathrm{kV}$.

Silica colloids with diameter of $492 \mathrm{~nm}$ and a polydispersity of $3.2 \%$ were synthesized according to the Stöber method. These particles were subsequently covered by a layer of 3-methacryloxypropyltrimethoxysilane using a method described by Philipse and Vrij. ${ }^{[19]}$

Colloidal crystals were grown by immersing a clean, 100-nmthick silicon nitride window into a $1 \mathrm{v} / \mathrm{v} \%$ aqueous dispersion of polystyrene colloids or a $0.2 \mathrm{v} / \mathrm{v} \%$ aqueous dispersion of silica colloids and slowly evaporating the solvent in an oven at $50^{\circ} \mathrm{C} .{ }^{[1]}$ The thin silicon nitride windows are almost X-ray transparent and, as such, prevent significant attenuation of the $\mathrm{X}$-ray signal by the substrate. 
STXM Imaging: Investigation of the polystyrene colloidal crystals by STXM was performed at beamline 11.0.2. of the Advanced Light Source synchrotron facility at the Lawrence Berkeley National Laboratory, California, USA. ${ }^{[20]}$ During the experiment the synchrotron operated at a $500 \mathrm{~mA}$ ring current in top-off mode $(1.9 \mathrm{GeV})$. Beamline 11.0.2 is a 5 -cm-period elliptical polarization undulator (EPU5) beamline with an accessible energy range of 100-2000 eV. For carbon K-edge imaging, the undulator first harmonic X-rays were irradiated on a 1200 lines $\mathrm{mm}^{-1}$ plane-grating monochromator to select the required photon energy between 278 and $315 \mathrm{eV}$. At these conditions, a flux of $\approx 5 \times 10^{12}$ photons s ${ }^{-1}$ was obtained. The silica colloidal crystals were studied with the STXM microscope at beamline 10ID-1 (SM) at the Canadian Light Source (CLS), University of Saskatchewan, Canada. ${ }^{[22]}$

A $240-\mu \mathrm{m}$-diameter zone plate (ZP) with a central stop of $95 \mu \mathrm{m}$ and an outermost zone width $\Delta r$ of $25 \mathrm{~nm}$ was used to focus the light with a Rayleigh-criterion spatial resolution $\Delta r_{\text {Rayleigh }}$ of $30 \mathrm{~nm}$ $\left(\Delta r_{\text {Rayleigh }} \approx 1.22 \cdot \Delta r\right)$. The ZP's central stop and an order-sorting aperture (OSA) were used to select the first-order diffracted $X$-rays for spectroscopy and imaging. The colloidal crystals on silicon nitride windows were mounted on a piezoelectric sample stage to translate the sample. As a result, the sample could be focused $(\Delta z)$ and raster scanned $(\Delta x, \Delta y)$. Transmitted light was detected by a scintillator screen combined with a photomultiplier tube (PMT). Typical images were acquired in a point-by-point mode with a $1 \mathrm{~ms}$ dwell time per pixel, a $5 \mu \mathrm{m} \times 5 \mu \mathrm{m}$ field of view (FOV), and a $10 \mathrm{~nm} \times 10 \mathrm{~nm}$ pixel size. Taking dead time between the acquisition of different pixels into account, the recording of a single transmission image typically took $6 \mathrm{~min}$.

\section{Supporting Information}

Supporting Information is available from the Wiley Online Library or from the author. It contains SEM images of the studied crystals, calculations of the linear X-ray absorption regime for thickness determination of silica-based crystals at the oxygen K-edge, and a detailed comparison of the lateral spatial resolution, axial resolving power, and penetration depth of CSLM, STXM, and SEM.

\section{Acknowledgements}

This work was financially supported by a VICI grant (FMFdG) of the Netherlands Organization for Scientific Research (NWO-CW). Sandy Heinen is acknowledged for polystyrene particle synthesis. We thank beamline 11.0.2. of the ALS and beamline 10ID-1 (SM) at the CLS for beam time and support. The ALS is supported by the Director, Office of Science, Office of Basic Energy Sciences, of the U.S. Department of Energy under Contract No. DE-ACO205CH11231. The CLS is supported by the Natural Sciences and Engineering Research Council of Canada, the National Research Council Canada, the Canadian Institutes of Health Research, the Province of Saskatchewan, Western Economic Diversification Canada, and the University of Saskatchewan.
[1] P. Jiang, J. F. Bertone, K. S. Hwang, V. L. Colvin, Chem. Mater. 1999, 11, 2132.

[2] A. Blanco, E. Chomski, S. Grabtchak, M. Ibisate, S. John, S. W. Leonard, C. Lopez, F. Meseguer, H. Miguez, J. P. Mondia, G. A. Ozin, O. Toader, H. M. van Driel, Nature 2000, 405, 437.

[3] Y. A. Vlasov, X. Z. Bo, J. C. Sturm, D. J. Norris, Nature 2001, 414, 289.

[4] D. J. Norris, E. G. Arlinghaus, L. Meng, R. Heiny, L. E. Scriven, Adv. Mater. 2004, 16, 1393.

[5] E. Yablonovitch, Sci. Am. 2001, 285, 47.

[6] J. E. G. J. Wijnhoven, W. L. Vos, Science 1998, 281, 802.

[7] R. Rengarajan, D. Mittleman, C. Rich, V. Colvin, Phys. Rev. E 2005, $71,016615$.

[8] L. Meng, H. Wei, A. Nagel, B. J. Wiley, L. E. Scriven, D. J. Norris, Nano Lett. 2006, 6, 2249.

[9] D. D. Brewer, J. Allen, M. R. Miller, J. M. De Santos, S. Kumar, D. J. Norris, M. Tsapatsis, L. E. Scriven, Langmuir 2008, 24, 13683.

[10] E. Vekris, V. Kitaev, D. D. Perovic, J. S. Aitchison, G. A. Ozin, Adv. Mater. 2008, 20, 1110.

[11] J. Hilhorst, V. V. Abramova, A. Sinitskii, N. Sapoletova, K. S. Napolskii, A. A. Eliseev, D. V. Byelov, N. A. Grigoryeva, A. V. Vasilieva, W. G. Bouwman, K. Kvashnina, A. Snigirev, S. V. Grigoriev, A. V. Petukhov, Langmuir 2009, 25, 10408.

[12] A. Van Blaaderen, P. Wiltzius, Science 1995, 270, 1177.

[13] H. Wei, L. Meng, Y. Jun, D. J. Norris, Appl. Phys. Lett. 2006, 89, 241913.

[14] T. A. Klar, S. Jakobs, M. Dyba, A. Egner, S. W. Hell, Proc. Natl. Acad. Sci. USA 2000, 97, 8206.

[15] A. V. Petukhov, D. G. A. L. Aarts, I. P. Dolbnya, E. H. A. De Hoog, K. Kassapidou, G. J. Vroege, W. Bras, H. N. W. Lekkerkerker, Phys. Rev. Lett. 2002, 88, 208301.

[16] J. H. J. Thijssen, A. V. Petukhov, D. C. 't Hart, A. Imhof, C. H. M. Van Der Werf, R. E. I. Schropp, A. van Blaaderen, Adv. Mater. 2006, 18, 1662.

[17] A. Bosak, I. Snigireva, K. S. Napolskii, A. Snigirev, Adv. Mater. 2010, 22, 3256.

[18] E. B. Mock, H. de Bruyn, B. S. Hawkett, R. G. Gilbert, C. F. Zukoski, Langmuir 2006, 22, 4037.

[19] A. P. Philipse, A. Vrij, J. Colloid Interface Sci. 1989, 128, 121.

[20] A. L. D. Kilcoyne, T. Tyliszczak, W. F. Steele, S. Fakra, P. Hitchcock, K. Franck, E. Anderson, B. Harteneck, E. G. Rightor, G. E. Mitchell, A. P. Hitchcock, L. Yang, T. Warwick, H. Ade, J. Synchrotron Radiat. 2003, 10, 125.

[21] H. Bluhm, K. Andersson, T. Araki, K. Benzerara, G. E. Brown, J. J. Dynes, S. Ghosal, M. K. Gilles, H. C. Hansen, J. C. Hemminger, A. P. Hitchcock, G. Ketteler, A. L. D. Kilcoyne, E. Kneedler, J. R. Lawrence, G. G. Leppard, J. Majzlam, B. S. Mun, S. C. B. Myneni, A. Nilsson, H. Ogasawara, D. F. Ogletree, K. Pecher, M. Salmeron, D. K. Shuh, B. Tonner, T. Tyliszczak, T. Warwick, T. H. Yoon, J. Electron Spectros. Relat. Phenom. 2006, $150,86$.

[22] K. V. Kaznatcheev, C. Karunakaran, U. D. Lanke, S. G. Urquhart, M. Obst, A. P. Hitchcock, Nucl. Instrum. Meth. Phys. Res. 2007, 582, 96.

[23] D. T. Attwood, Soft X-rays and Extreme Ultraviolet Radiation, 1st Ed., Cambridge University Press, Cambridge, UK 2007.

[24] Y. Vladimirsky, D. P. Kern, T. H. P. Chang, D. T. Attwood, N. Iskander, S. Rothman, K. McQuaide, J. Kirz, H. Ade, I. McNulty, H. Rarback, D. Shu, Nucl. Instrum. Meth. Phys. Res. 1988, 266, 324.

[25] F. de Groot, Chem. Rev. 2001, 101, 1779.

[26] F. de Groot, Coord. Chem. Rev. 2005, 249, 31.

[27] aXis2000 is free for noncommercial use. It is written in Interactive Data Language (IDL) and is available online: http://unicorn. mcmaster.ca/aXis2000.html.

[28] B. L. Henke, E. M. Gullikson, J. C. Davis, Atomic Data Nucl. Data Tables 1993, 54, 181. 
[29] W. Wang, S. A. Asher, J. Am. Chem. Soc. 2001, 123, 12528.

[30] J. Kikuma, B. P. Tonner, J. Electron Spectros. Relat. Phenom. 1996, 82, 53.

[31] M. Taillefumier, D. Cabaret, A.-M. Flank, F. Mauri, Phys. Rev. B 2002, 66, 195107.

[32] M. E. Leunissen, C. G. Christova, A.-P. Hynninen, C. P. Royall, A. I. Campbell, A. Imhof, M. Dijkstra, R. van Roij, A. van Blaaderen, Nature 2005, 437, 235.

[33] M. P. Seah, W. A. Dench, Surf. Interface Anal. 1979, 1, 2.

[34] R. H. Webb, Rep. Progr. Phys. 1996, 59, 427.

[35] R. F. Egerton, Physical Principles of Electron Microscopy, 3rd Ed., Springer, New York, USA 2008.

[36] W. Chao, B. D. Harteneck, J. A. Liddle, E. H. Anderson, D. T. Attwood, Nature 2005, 435, 1210

[37] A. P. Hitchcock, J. Li, S. R. Reijerkerk, P. Foley, H. D. H. Stöver, I. Shirley, J. Electron Spectros. Relat. Phenom. 2007, 156-158, 467.

[38] G. A. Johansson, T. Tyliszczak, G. E. Mitchell, M. H. Keefe, A. P. Hitchcock, J. Synchrotron Radiat. 2007, 14, 395.

[39] H. Friedrich, C. J. Gommes, K. Overgaag, J. D. Meeldijk, W. H. Evers, B. de Nijs, M. P. Boneschanscher, P. E. de Jongh, A. J. Verkleij, K. P. de Jong, A. van Blaaderen, D. Vanmaekelbergh, Nano Lett. 2009, 9, 2719.
[40] S. Jeong, L. Hu, H. R. Lee, E. Garnett, J. W. Choi, Y. Cui, Nano Lett. 2010, 10, 2989.

[41] M. M. van Schooneveld, V. W. A. de Villeneuve, R. P. Dullens, D. G. A. L. Aarts, M. E. Leunissen, W. K. Kegel, J. Phys. Chem. B 2009, 113, 4560.

[42] M. C. Mourad, E. J. Devid, M. M. van Schooneveld, C. Vonk, H. N. W. Lekkerkerker, J. Phys. Chem. B 2008, 112, 10142.

[43] J. F. Creemer, S. Helveg, G. H. Hoveling, S. Ullmann, A. M. Molenbroek, P. M. Sarro, H. W. Zandbergen, Ultramicroscopy 2008, 108, 993.

[44] E. de Smit, I. Swart, J. F. Creemer, G. H. Hoveling, M. K. Gilles, T. Tyliszczak, P. J. Kooyman, H. W. Zandbergen, C. Morin, B. M. Weckhuysen, F. M. F. de Groot, Nature 2008, 456, 222.

[45] E. de Smit, I. Swart, J. F. Creemer, C. Karunakaran, D. Bertwistle, H. W. Zandbergen, F. M. F. de Groot, B. M. Weckhuysen, Angew. Chem. Int. Ed. 2009, 48, 3632.

[46] B. M. Weckhuysen, Angew. Chem. Int. Ed. 2009, 48, 4910.

[47] F. M. F. de Groot, E. de Smit, M. M. van Schooneveld, L. R. Aramburo, B. M. Weckhuysen, ChemPhysChem 2010, 11, 951.

Received: October 3, 2010

Published online: February 18, 2011 